\title{
THE EFFICACY OF SOME VOLATILE OIL EXTRACTS AS OLFACTORY STIMULI TO THE FRUIT FLIES, Bactrocera zonata (Saunders) AND Ceratitis capitata (Wiedemann) (Diptera : Tephritidae) \\ Abd El-Kareim, A. I*; L. M. Shanab*; M. E. El-Naggar** and N. M. A. Ghanim ** \\ * Economic Entomology Department, Faculty of Agriculture, Mansoura University \\ ** Plant Protection Research Institute, Agricultural Research Center, Ministry of Agriculture
}

\begin{abstract}
The experiments were carried out in guava orchard at Mansoura district, Dakahlia governorate, Egypt. Eight different plant oil extracts [olive, orange, peppermint, basil, clove (Egyptian \& French), parsley and black cumin] were bioassayed as attractants for the tephritids, $B$. zonata and $C$. capitata males and females. $B$. zonata males and females as well as $C$. capitata females showed no response to all the tested oils. C. capitata males exhibited a significantly positive response to French and Egyptian clove oils; while it had no response to olive, basil, parsley and black cumin oils. The residual effectiveness of Egyptian and French clove oils were lost after nine days of exposure.

Comparative study between prepared (local) and imported methyl eugenol was evaluated for 72 successive days. Statistical analysis indicated that there were significant differences between the local and imported methyl eugenol after 6, 12 and 18 days only. So, the local methyl eugenol traps may be used as a valuable tool in monitoring $B$. zonata.

The attractiveness of $B$. zonata males to felt and plant fibers blocks (with different thickness) saturated with methyl eugenol was evaluated. The felt blocks captured the highest number of $B$. zonata males in comparison with those of plant fibers. The efficiency of plant fiber blocks was significantly affected by block thickness; while felt blocks did not affected by thickness.
\end{abstract}

\section{INTRODUCTION}

Fruits are considered as one of the most important crops in the whole world because of their nutritional and economical importance as well as for local consumption or exportation. B. zonata and C. capitata (Order: Diptera, Family: Tephritidae) are the most serious pests of fruits. Their females lay their eggs in the fruits and the hatching maggots devour into the pulp. Subsequently, secondary infestations with bacterial and fungal diseases mostly exist and the infested fruits drop down (White and Elson-Harris, 1994).

In Egypt, B. zonata became a serious pest in the last decade attacking a wide range of fruits that differ in their ripening time stage all over the year. It is a polyphagus pest attacking 40 species of fruit and vegetable crops as well as wild host plants (Syed et al., 1970).

Semiochemicals are defined as "substances which transmit massages between living organisms, both plant and animals" (Law and 
Abd El-Kareim, A. l. et al.

Regnier, 1971). The males of several economically important tephritid species are strongly attracted to particular chemical compounds, termed "male lures" or "parapheromones", that either occur naturally in plants or (presumed) synthetic analogues or plant-borne substances (Sivinski \& Calkins, 1986 and Fletcher, 1987).

"Male lures" are used in the monitoring of $B$. zonata and $C$. capitata populations. According to Afia (2007), methyl eugenol could be used in male annihilation technique of $B$. zonata eradication or control programs.

So, the present work aimed to evaluate the reaction behavior of $B$. zonata and $C$. capitata (males and females) in response to some plant oil extracts. Also, comparative studies were done to compare the attractiveness of $B$. zonata males to the prepared (local) and imported methyl eugenol. Additionally, to evaluate the influence of block type on the potentiality of methyl eugenol in relation to the population of $B$. zonata males.

\section{MATERIALS AND METHODS}

The experiments were carried out in an area of about two feddans cultivated with guava (Psidium guajava) in the experimental farm of Faculty of Agriculture, Mansoura University at Mansoura district, Dakahlia governorate, Egypt.

\section{1- Evaluation the attractiveness of $B$. zonata and $C$. capitata adults to some plant oil extracts :}

To evaluate the efficacy of eight plant oil extracts [olive, orange, peppermint, basil, clove (Egyptian and French), parsley and black cumin] as attractants for males and females of $B$. zonata and $C$. capitata, bioassay was carried out in McPhail traps (McPhail et al., 1963). About $100 \mathrm{ml}$ of the extract were put in each trap. Each extract was replicated five times. The traps were distributed in a completely randomized design during September 2006. After three days, the traps were tested and the number of attracted males and females of each species were counted and recorded.

To estimate the residual activity of Egyptian and French clove oils in attracting C. capitata males, sex sticky traps (Jackson traps) were used. Every five traps (as replicates) were powered with one of the tested oil (by saturating a piece of cotton dispenser with the oil). All the prepared traps were distributed in a completely randomized design. Captured males were collected every three days throughout 21 successive days (from the $3^{\text {rd }}$ till $24^{\text {th }}$ of October 2006) and numbers of $C$. capitata males were counted and recorded to estimate the capture/trap/day (CTD).

2- Comparative efficacy of the prepared (local) and imported methyl eugenol in attracting $B$. zonata males :

2-1- Preparing the local methyl eugenol :

Isolation and methylation of eugenol were done according to (Youssef, 1997). 
Isolation of eugenol :

The volatile oil was extracted from clove (Eugenia caryophyllus) by steam distillation. This oil is the essential oil contains eugenol in $98 \%$ and caryophyllene derivatives (sesquiterpenes) non-phenolic about 2\%. Eugenol was isolated by treatment of the extracted oil with an aqueous sodium hydroxid solution (3-5\%). The non-phenolic constituent was extracted with chloroform. The aqueous alkaline solution was acidified with dilute hydrochloric acid to give eugenol, which was obtained by distillation.

\section{Methylation of eugenol by dimethyl sulphate :}

A solution of eugenol $(2 \mathrm{gm})$ in acetone $(8 \mathrm{ml})$ and in the presence of sodium hydroxide $(20 \mathrm{ml}, 56 \%)$ was stirred on ice bath. During the stirring, dimethyl sulphate $(2 \mathrm{ml})$ was added dropwise to the reaction mixture which was stirred for 6 hours, then poured on ice cold water. Methyl eugenol ether was extracted with chloroform $(3 \times 20 \mathrm{ml})$, washed with water $(3 \times 20 \mathrm{ml})$ and dried over anhydrous sodium sulphate. The solvent $\left(\mathrm{CHCl}_{3}\right)$ was evaporated under reduced pressure to give methyl eugenol ether as a pall yellow oil (1.604 gm, $80 \%$ yield).

\section{2-2- Field bioassay :}

Jackson traps powered with prepared (local) or imported methyl eugenol were used. Each treatment was replicated five times and distributed in a completely randomized design. Traps were tested every six days (as an interval period) and the numbers of captured males of $B$. zonata were recorded to estimate the CTD. This experiment was carried out during the period from the $3^{\text {rd }}$ of October till 14th of December 2006 (72 days).

\section{3- Estimate the influence of block type on the potentiality of methyl eugenol as bio-control agent against $B$. zonata males :}

This experiment was conducted in the previously mentioned orchard during the period from the $13^{\text {th }}$ of September till the $21^{\text {st }}$ of November 2007.

\section{The experimental blocks :}

Two types of blocks, felt and plant fibers $\left(25 \mathrm{~cm}^{2}\right)$ were used as dispensers for methyl eugenol. For each block type, two thicknesses ( $3 \& 6$ $\mathrm{mm}$ for felt and $6 \& 11 \mathrm{~mm}$ for plant fiber blocks) were also used for bioassay.

All of the tested blocks were dipped in a mixture of $80 \%$ methyl eugenol and $20 \%$ pure malathion for about four hours in the laboratory. After that, the blocks were transferred to the field. Each block type and thickness was replicated five times and distributed in a completely randomized design.

To collect the dead insects, a plastic bag fixed with an iron ring (40 $\mathrm{cm}$ in diameter) was put under the block for receiving the dead male flies.

The captured flies in the plastic bags were investigated every five days (as an interval period) and the numbers of $B$. zonata males were counted and recorded.

Statistical analysis was done as one way ANOVA and L.S.D. values were estimated at the probability of $5 \%$. In addition, the simple regression analysis was calculated. 
Abd El-Kareim, A. I. et al.

\section{RESULTS}

1- Evaluation the attractiveness of $B$. zonata and $C$. capitata (males \& females) in response to some plant oil extracts :

Eight different plant oil extracts were bioassayed as attractants for the tephritid adults, $B$. zonata and $C$. capitata (male and females). The obtained data as illustrated in Table (1) obviously indicated that females of both $B$. zonata and $C$. capitata not showed any response to any of the tested oils.

Table (1): Capture/trap/day (CTD) of B. zonata (PFF) and C. capitata (MFF) males captured by different plant oil extracts in McPhail traps after 3 days in guava orchards at Mansoura district.

\begin{tabular}{|l|c|c|c|c|}
\hline \multirow{2}{*}{ Oil extract } & \multicolumn{4}{c|}{ After 3 days } \\
\cline { 2 - 5 } & \multicolumn{2}{|c|}{ PFF } & \multicolumn{2}{c|}{ MFF } \\
\cline { 2 - 5 } & Females & Males & Females & Males \\
\hline Olive & 0.0 & 0.0 & 0.0 & 0.0 \\
Orange & 0.0 & 0.0 & 0.0 & 0.67 \\
Peppermint & 0.0 & 0.0 & 0.0 & 0.13 \\
Basil & 0.0 & 0.0 & 0.0 & 0.0 \\
French clove & 0.0 & 0.0 & 0.0 & 24.33 \\
Egyptian clove & 0.0 & 0.0 & 0.0 & 6.13 \\
Parsley & 0.0 & 0.0 & 0.0 & 0.0 \\
Black cumin & 0.0 & 0.0 & 0.0 & 0.0 \\
\hline
\end{tabular}

As shown in Table (1), males of $B$. zonata showed no response to the tested plant oil extracts, while $C$. capitata males exhibited a significantly positive response to French and Egyptian clove oils (CTDs were 24.33 and 6.13). The obtained data also illustrated that $C$. capitata males had a low attractiveness to orange $(C T D=0.67)$ and peppermint $(C T D=0.13)$ oils, while $C$. capitata males showed no response to the other tested oils (olive, basil, parsley and black cumin) (Table, 1).

From the previously mentioned results, it could be concluded that clove oil can be used as a "male lure" for $C$. capitata males.

\section{2- Residual activity of Egyptian and French clove oils in attracting $C$. capitata males :}

Data represented in Figure (1) cleared that French and Egyptian clove oils lost their activity after nine days of exposure. The regression analysis obviously indicated that French clove oil lost its activity with an approximately higher rate of attraction than that of Egyptian one. However, the slope of regression line was -0.57 and -0.10 for French and Egyptian clove oils. 


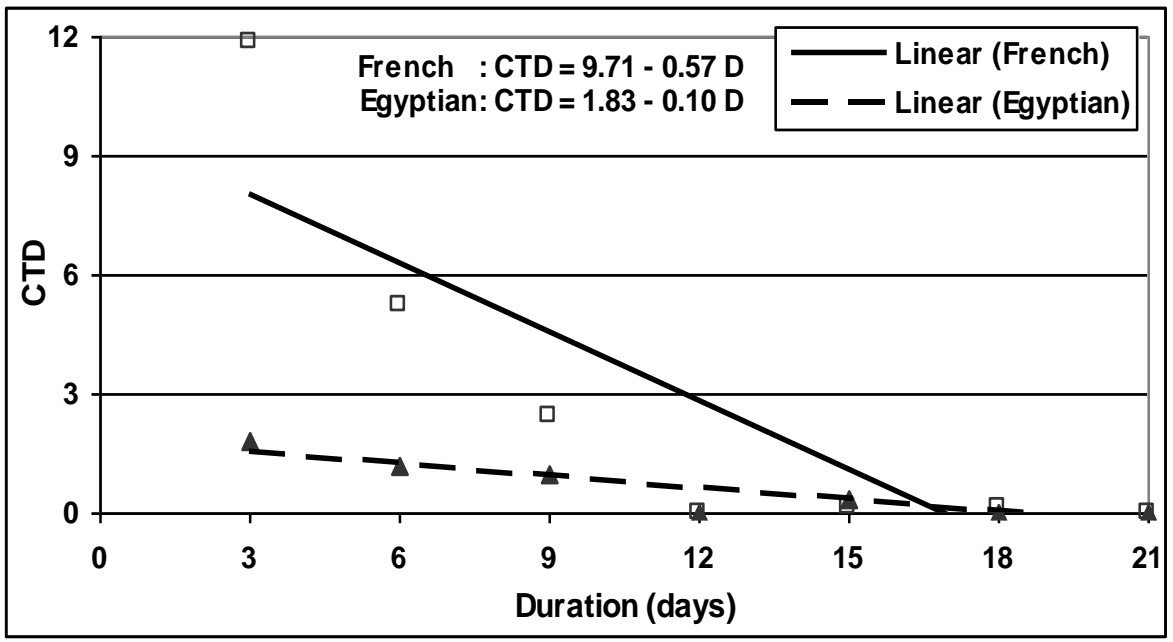

Figure (1): Residual activity of Egyptian and French clove oils against $C$. capitata males in guava orchards at Mansoura district.

As shown in Figure (1), at the beginning of exposure, the efficacy of French clove oil was significantly higher than the Egyptian one. The attractiveness power of them decreased gradually till the end of the experiment (21 days).

It could be concluded that, French clove oil extract could be used as male lure for $C$. capitata but with renewing it every one weak.

So, these results demonstrate the potential of French eugenol oil as a cheaper alternative attractant in monitoring $C$. capitata population.

3- Comparative efficacy of the prepared (local) and imported methyl eugenol in attracting $B$. zonata males :

The effectiveness of local and imported methyl eugenol was evaluated at 6 days interval by using Jackson traps under field conditions during the period from the $3^{\text {rd }}$ of October till $14^{\text {th }}$ of December 2006 (72 days).

The attractiveness of $B$. zonata males in response to both chemicals was illustrated in Figure (2). The CTD of local methyl eugenol after 6 days was 3.80 then increased to reach 35.50 after 24 days. After that the CTD has been decreased gradually till the end of the experiment, however the CTD reached 10.80 (after 72 days). 
Abd El-Kareim, A. l. et al.

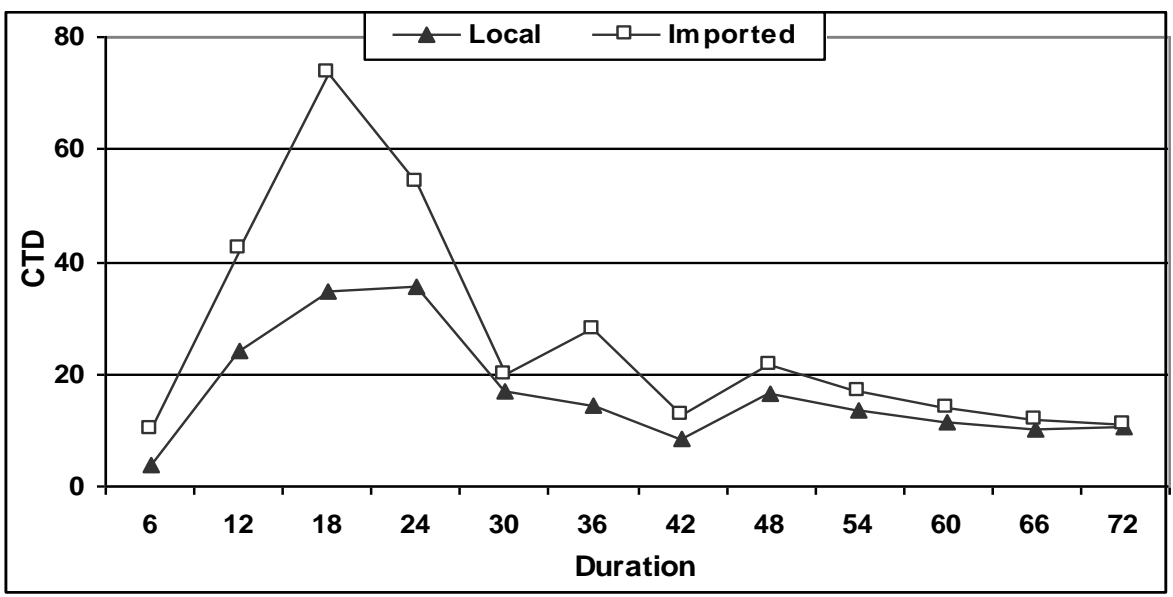

Figure (2): Fluctuations in numbers of $B$. zonata males in Jackson traps baited with local and imported methyl eugenol over 72 days in guava fields at Mansoura district (without renewal the attractants).

As shown in Figure (2), B. zonata males exhibited high attraction to the imported methyl eugenol than the local one. However, the CTD was 10.10 after 6 days and increased to reach 73.50 after 18 days. Then the CTD decreased gradually till reach 11.00 at the end of the experiment (after 72 days).

Statistical analysis indicated that there were significant differences between the local and imported methyl eugenol after 6,12 and 18 days only.

So it could be concluded that the prepared (local) methyl eugenol may be used in monitoring $B$. zonata population.

4- Influence of block type on the efficiency of methyl eugenol as biocontrol agent against $B$. zonata :

The attractiveness of $B$. zonata males to block types (felt and plant fibers) with different thickness saturated with methyl eugenol was evaluated and illustrated in Figure (3).

The number of captured males / block / five days for each block type was fluctuated over the tested period (70 days). The efficiency of the tested block types was generally higher during the second month than the first one. The highest capture of $B$. zonata males in felt $(6 \& 3 \mathrm{~mm})$ and plant fiber blocks ( $11 \& 6 \mathrm{~mm})$ were recorded after $(40 \& 35)$ and 55 days, respectively (Figure, 3). 


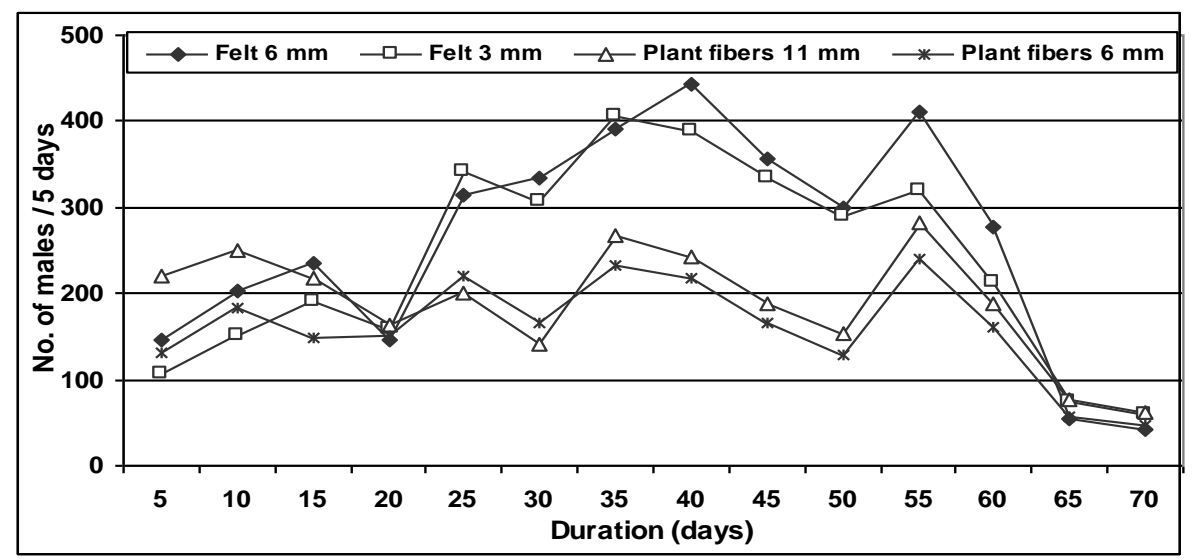

Figure (3): Fluctuation of PFF males captured by felt $(6 \& 3 \mathrm{~mm})$ and plant fiber (11 \& $6 \mathrm{~mm}$ thickness) blocks saturated with a mixture of $80 \%$ methyl eugenol and $20 \%$ pure malathion over $\mathbf{7 0}$ days in guava fields at Mansoura district (without renewal the attractant).

As shown in Figure (3), the highest capture of $B$. zonata males was recorded on felt blocks in comparison with plant fiber ones. The mean numbers for all the investigation period of captured males / block / five days were $260.9,238.3,189.1$ and 160.6 males for felt $(6 \& 3 \mathrm{~mm})$ and plant fibers (11 \& $6 \mathrm{~mm}$ thickness), respectively. Statistical analysis indicated that there were significant differences between block types of felt and plant fibers (L.S.D.P=5\% $=26.8$ ).

In respect to block thickness, statistical analysis obviously indicated that the efficiency of plant fibers was significantly affected by block thickness; while, the efficiency of felt blocks did not affected by thickness.

\section{DISCUSSION}

The present results indicated that some plant oil extracts (clove and orange) act as male lures for $C$. capitata adults. Species of Dacine basically respond more to olfactory cause than visual cause (Drew and Lioyed, 1987). The males of Bactrocera and Ceratitis may be collected in trap that has been baited with chemical attractants, which are sometimes referred to as Parapheromones (Allwood et al., 1999). Some workers believe that the male lures are Kairomones, which are behavior-modifying plant chemicals.

In the present study, C. capitata males were attracted to clove oil extracts more than the other tested oil extracts. This may be attributed to the presence of methyl eugenol in the clove oil extracts. Also, Tan (1993 \& 2000) reported that males of $B$. dorsalis (of the tephritid fruit flies) feed voraciously on methyl eugenol, a component of plant essential oil found in at least 200 species of plants from 32 families.

The methyl eugenol group consists of methyl eugenol, citronella oil and huon pine oil of citronella oil (Allwood et al., 1999). Methyl eugenol attracts males of many Bactrocera spp. and it attracts some of subgenus 
Abd El-Kareim, A. l. et al.

Ceratitis. Under recent chemical nomenclature it called methoxy eugenol and it has been known as eugenol methyl ether (Drew, 1974 \& 1982).

Chemical and behavioral studies showed that methyl eugenol is converted to phenylpropanoids-DMP that functions as male sex pheromonal components (Nishida et al., 1988; Tan \& Nishida, 1996 \& 1998 and Hee \& Tan, 1998). Also, Hee \& Tan (2004 \& 2005) reported the presence of these pheromonal components in the hemolymph of $B$. dorsalis males fed on methyl eugenol, which suggests the hemolymph involvement in the transport of attractant-derived pheromonal components from the crop to the rectal gland organ where the compounds are released during dusk.

The present study revealed that the imported methyl eugenol had a higher efficacy in attracting $B$. zonata males than the local one. Statistically, there were significant differences between the local and imported methyl eugenol after 6, 12 and 18 days only, while there were insignificant differences between them during the rest of the tested period (72 days).

Hanafy et al. (1999) (in Egypt) and Gopaul \& Price (1999) (in Mauritius) found locally produced food attractants as a cheaper alternative attractants for fruit flies. Also, in the present study, the local methyl eugenol may be as a cheaper alternative attractant by using some chemical operations for using in $B$. zonata population monitoring.

Methyl eugenol was successfully used in combination with several different insecticides (such as malathion or dichlorvos or naled with as poison baits) for detecting and surveying the male flies of $B$. zonata in orchards (Mitchell et al., 1985; Grewal \& Kapoor, 1987 and Gupta et al., 1990). So, methyl eugenol can either be easily used as a standard attractant to control fruit flies.

The present study revealed that felt blocks were more suitable than the plant fiber blocks for male lures. Also, Afia (2007) found that felt dispenser was one of the highest tested dispensers for male lure (with methyl eugenol) of $B$. zonata. This may be attributed to the higher initial of release rate of male lure in plant fiber blocks than felt ones; which losses the attractiveness of plant fiber rapidly. This explanation come in the same line with that obtained by Afia (2007).

\section{REFERENCES}

Afia, Y.E. (2007). Comparative studies on the biology and ecology of the two fruit flies in Egypt, Bactrocera zonata (Saunders) and Ceratitis capitata (Wiedemann). Ph. D. Thesis, Faculty of Agriculture, Cairo University, $301 \mathrm{pp}$.

Allwood, A. L.; A. Chinajariyawong; R. A. I. Drew; E. L. Hamacek; D. L. Hancock; C. Hengsawad; J. C. Jipanin; M. Jirasurat; K. C. Kong; S. Kritsaneepaiboon; C. T. S. Leong and S. Vijaysegaran (1999). Host plant records for fruit flies (Diptera: Tephritidae) in South east Asia. Roffles Bull. of Zoology, Supplement, 7: 18-19.

Drew, R. A. I. (1974). The response of fruit fly species (Diptera: Tephritidae) in the South Pacific area to male attractants. J. Australian Entomol. Soc., 13: 267-270. 
Drew, R. A. I. (1982). Fruit fly collecting. In: Economic fruit flies of the South Pacific Region (Ed. by Drew, R. A.; G. H. S. Hooper and M. A. Bateman) (2nd edition), PP. 129-139. Queensland Department of Primary Industries, Brisbane, Australia.

Drew, R. A. I. and A. C. Lioyed (1987). Relationship of fruit flies (Diptera: Tephritidae) and their Bactrocera spp. to host plants. Ann. Ent. Soc. Am., 80: 629-636.

Fletcher, B. S. (1987). Temperature development rate relationships of the immature stages and adult of tephritid fruit flies. Pp 273-289 in Robinson, A. S. and G. Hooper (Eds.) fruit flies their biology, natural enemies and control. World crop pests 3A. Amsterdam, Elsevier.

Gopaul, S. and N. S. Price (1999). Local production of protein bait for use in fruit fly monitoring and control. Food and Agricultural Research Council, Réduit, Mauritius, 117-122.

Grewal, J. S. and V. C. Kapoor (1987). A new collapsible fruit fly trap. J. Ent. Res., 11 (2): 203-206.

Gupta, D.; A. K. Verma and O. P. Bhalla (1990). Population of fruit flies (Dacus zonatus and $D$. dorsalis) infesting fruit crops in north Western Himalayan region. India J. of Agric. Sci., 60 (7): 471- 474.

Hanafy, A. H.; Kh. M. Moursi and M. S. Al-Shahaat (1999). Filed evaluation of the attractability of local food attractants for the olive fruit fly, Bactrocera oleae (Gmelin) in the Egyptian Western Desert. $2^{\text {nd }}$, Int. Conf. of Pest Control, Mansoura, Egypt, 119-128pp.

Hee, A. K. W. and K. H. Tan (1998). Attraction of female and male Bactrocera papayae conspecific males fed with methyl eugenol and attraction of females to male sex pheromone components. J. Chem. Ecol., 24: 753764.

Hee, A. K. W. and K. H. Tan (2004). Male sex pheromone components derived from methyl eugenol in the hemolymph of the fruit fly Bactrocera papayae. J. Chem. Ecol., 30: 2127-2138.

Hee, A. K. W. and K. H. Tan (2005). Bioactive fractions containing methyl eugenol-derived sex pheromonal components in hemolymph of the male fruit fly Bactrocera dorsalis (Diptera: Tephritidae). Bull. Entomol. Res., 95: 615-620.

Law, J. H. and Regnier (1971). Pheromones. Annual Review of Biochemistry 40: 533-548.

McPhail, M.; F. D. Lopez and J. F. M. Velasco (1963). Within package ethylene dibromide fumigations on mangoes and grapefruit in fiberboard cartons to destroy Mexican fruit fly infestation. J. Econ. Entomol., 56 (3): 496.

Mitchell, W. C.; R. L. Metcalf; E. R. Metcalf and S. Mitchell (1985). Candidate substitutes for methyl eugenol as attractants for the area wide monitoring and control of the Oriental fruit fly Dacus dorsalis Handel. (Diptera: Tephritidae). Environ. Entomol., 14: 171-181.

Nishida, R.; K. H. Tan; M. Serit; N. H. Lajis; A. M. Sukari; S. Takahashi and $\mathrm{H}$. Fukami (1988). Accumulation of phenylpropanoids in the rectal glands of males of the Oriental fruit fly Dacus dorsalis. Experentia 44: 534-536. 
Sivinski, J. M. and C. Calkins (1986). Pheromones and Parapheromones in the control of tephritids. Florida Entomol., 69: 157-168.

Syed, R. A.; M. A. Ghani and M. Murtaza (1970). Studies on the tephritids and their natural enemies in west Pakistan. III. Dacus zonatus (Saunders) (Diptera: Tephritidae). Tech. Bull. Comm. Wel. Inst. Biol. Cont., 13: 1-6.

Tan, K. H. (1993). Ecohormones for the management of fruit fly pests---understanding plant---- fruit---- fly---- predator interrelationships. Management of Insect Pests: Nuclear and Related Molecular and Generic Techniques. IAEA-SM, vol., 327. IAEA, Vienna, pp. 495-503.

Tan, K. H. (2000). Behaviour and chemical ecology of Bactrocera flies. In: Tan, K. H. (Ed.), Area-wide Control of Fruit Flies and Other Insect Pests. Penerbit Universiti Sains Malaysia, Penang, pp. 647-656.

Tan, K. H. and R. Nishida (1996). Sex pheromone and mating competition after methyl eugenol consumption in the Bactrocera dorsalis complex. In: McPheron, B.A. and G.J. Steck (Eds.), Fruit Fly pests. St. Lucie Press, Florida, 147-153pp.

Tan, K. H. and R. Nishida (1998). Ecological significance of male attractant in the defense and mating strategies of the fruit fly pest Bactrocera papayae. Entomol. Exp. Appl., 89: 155-158.

White, I. M. and M. M. Elson-Harris (1994). Fruit flies of economic significance: their identification and bionomics. $C A B$ International with ACIAR. p 601 + addendum.

Youssef, N. E. (1997). Chemical and photochemical studies on eugenol. M. Sc. Thesis, Faculty of Science, Mansoura University.

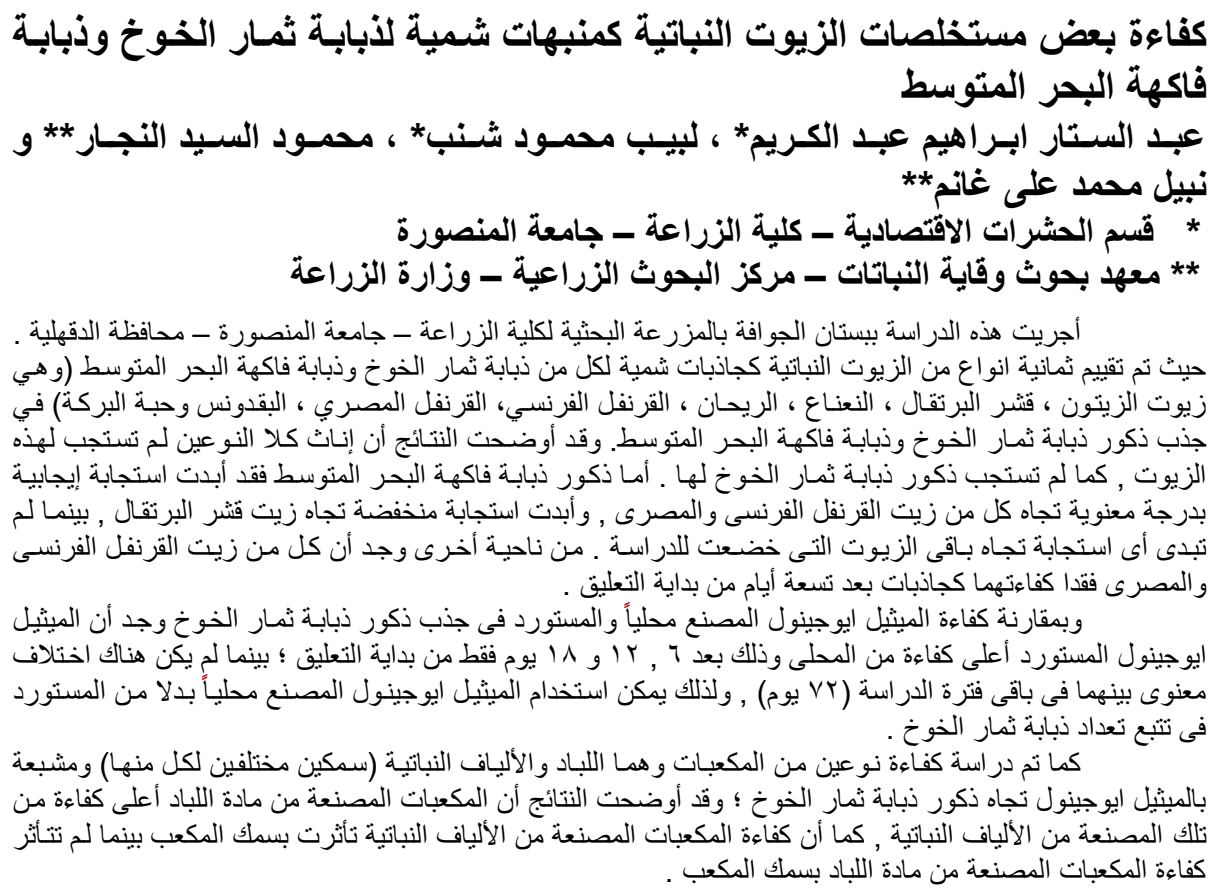

